\title{
EMG - supported biofeedback training in a person with multiple sclerosis - a case study
}

\begin{abstract}
Background: Until now, the etiology of Multiple Sclerosis (MS) has not been clarified. The symptoms vary from person to person and can affect motor, sensory, and cognitive body functions.

Objectives: Next to pharmacological treatments, non-pharmacological therapy approaches become more and more important for maintaining physical, sensory, psychological and social functions in people with MS (pwMS). Furthermore, Biofeedback (BF) seems to be an interesting treatment option in order to improve motor functions such as postural stability, walking safety and walking speed in pwMS
\end{abstract}

Methods: In the present case study, an Electromyography-Biofeedback (EMG-BF) has been examined over 6 weeks in a 74-year old female with MS. Functional Gait Assessment (FGA) and 10-Meter-Walking- Test (10MWT) has been tested pre (t0), after three weeks, (t1) and after six weeks ( $\mathrm{t} 2)$ of treatment $(2 \mathrm{x} /$ week).

Results: The results showed an increase in walking speed after 3 and 6 weeks in 10MWT and a decrease of postural stability after 3 weeks. In contrast, postural stability increased after 6 weeks. The participant tolerated the EMG-BF training very well and she felt more secure in everyday life in relating to a better perception of her feet in the moment of foot contact while walking.

Conclusions: EMG-BF training has been examined especially in order to improve the control of pelvic floor muscles in pwMS. Additionally, pwMS might benefit from BF training in regard to improve gait and postural control. More research is necessary to examine BF therapy options in order to support pwMS in their current motor impairments.
Volume 7 Issue 7 - 2017

\author{
Stephanie Kersten,' Britta Fuchs, ${ }^{2}$ Magnus \\ Liebherr ${ }^{3}$ \\ 'Hochschule Fresenius, Faculty of Health \& Social Sciences, \\ Germany \\ ${ }^{2}$ Carl Remigius Medical School, Germany \\ ${ }^{3}$ University Duisburg-Essen, General Psychology: Cognition, \\ Germany
}

Correspondence: Stephanie Kersten, Hochschule Fresenius, Faculty of Health \& Social Sciences, Germany, Limburgerstr. 2, 655I Idstein, Germany, Tel +49 (0)6126 - 9532 - 932 Email stephanie.kersten@hs-fresenius.de

Received: November 29, 2017 | Published: December 21 , 2017

\section{Introduction}

Multiple Sclerosis (MS) is defined as a "chronic, inflammatory, demyelinating disease of the central nervous system (CNS) with additionally differently pronounced loss of axons and reactive gliosis". ${ }^{1}$ Although etiological and pathogenetic processes are still unclear, it is assumed that MS can be assigned to autoimmune diseases, in which body tissue is attacked by the immune system. ${ }^{1,2}$ Due to their different inflammatory origin, persons with MS still differ in the manifestation of individual symptoms. The most common symptoms comprise sensory disturbances (approx. $40 \%$ of the patients), motor impairments (ca. 39\% of the patients), pain syndrome (ca. $15 \%$ of the patients), and cognitive impairments (ca. $10 \%$ of the patients). Therefore, MS must be seen as a complex and inter-individually strong varying neurodegenerative disease. On a symptomatic level, limitations can occur in neuropsychological functions (e.g. cognition, fatigue, depression), vegetative functions (e.g. bladder, dysentery, and sexuality disorders), as well as in deficits associated with the cerebral nerves (e.g. eye movement disorders, dysarthria, dysphagia) and pain phenomena. ${ }^{1,4}$ Sustained motor impairments are caused by spasticity, muscle weakness, gait and balance disorders. ${ }^{1,4}$ Significant differences in people with MS (pwMS) base on the fact that the course of the disease pattern is unpredictable. Until this day and age, pharmacological treatments are considered as gold standard in MS therapy. Thereby, pharmacological as well as non-pharmacological interventions must be seen as symptom-based treatment. Until now, the etiology of MS has not been clarified; in consequence it is absolutely necessary to further develop existing rehabilitation programs in order to maintain physical, sensory, psychological and social functions. In this context, first investigations pointed out the possibilities of using bio- and neurofeedback as a therapeutical intervention in this group of patients.
In biofeedback (BF) therapy, body signals are reported to the patients in real-time, so that the person can learn to influence these body functions. ${ }^{5}$ The aim of biofeedback treatment is the perception and influence of physical processes, which are important in the maintenance of mental, psychosomatic and physical diseases. Biofeedback can be used as a specific intervention, without already known side effects. ${ }^{5}$ For example, Jensen et al., ${ }^{6}$ used Neurofeedback (EEG-Biofeedback) to treat chronic pain in people with MS (pwMS). The authors reported a positive influence of the additional use of neurofeedback on selfhypnosis in chronic pain of pwMS. Choobforousshazadeh et al., investigated the effects of neurofeed back training on depressive symptoms and fatigue in pwMS. Furthermore, Lucio \& colleagues ${ }^{8}$ found an improved control of pelvic floor muscles in pwMS who suffer from sexual dysfunctions, after EMG-supported biofeedback training and additional neuromuscular electrostimulation. Prosperini et al., ${ }^{9}$ focused on the effects of visual-sensomotor biofeedback training (BFT) on postural control and the risk of falling in pwMS. Patients were asked to stand on a balance board, while visual feedback of their own performance were displayed on a monitor in front of them. The authors reported a significant increase in postural stability as well as a significant reduction of falls after a six week training period. ${ }^{9}$ Furthermore, participants showed improvements of visual, sensorimotor and vestibular compensation strategies after the six weeks. ${ }^{9}$ The possible applications of biofeedback and neurofeedback training are manifold and can be individually adapted to persons and health restrictions. Thereby, the comparability of individual investigations is difficult because of the wide range of experiments. In the present article a BFT should be applied and systematically examined in a person with MS in order to improve postural stability, walking safety as well as walking speed. Within the case study at hand, 
we apply an EMG-supported BFT, as a cost-effective and practicable method for everyday clinical practice.

\section{Methods}

\section{Anamnesis}

Inclusion criteria for participation in the study were as follows: (a) Confirmed MS diagnosis; b) regular participation in the BFT (2x/ week, maximum one missed date allowed); (c) limitations in postural stability; (d) complete testing at all three test times $\mathrm{T}_{0}, \mathrm{~T}_{1}, \mathrm{~T}_{2}$; (e) no acute cardiovascular disease, no acute orthopedic diseases and no other neurological disease.

The participant was a 74-year-old woman with a diagnosed MS and restricted postural stability. The anamnesis revealed a subjective insecurity while walking, and a "cuff feeling" around the ankle joints after prolonged standing or walking (regardless of the walking distance). The gait instability increases with large steps or overcoming obstacles (e.g. getting in and out of trains). Stair climbing is independently possible with railings. As a secondary diagnosis, the patient reported a conservatively treated Weber B fracture in the right lower limb (August 2016), a surgically treated humerus fracture of the right side (2003), medically well-adjusted hypertension and type II diabetes mellitus, as well as temporal knee and lumbar spine pain due to age-related degenerations. The MS was diagnosed in 1984 after two propulsions. Since then, a benign course has been shown in the form of sensory disturbances and stability impairment in the left lower limb and ankle joint area without further relapses $($ EDSS $=3.5)$. Due to the existing complaints, the patient is in regular physiotherapeutic and osteopathic treatment. The patient does not take any MS-specific drugs.

\section{Study protocol}

The present case study examines the effect of a six week BFT on improving postural stability, gait safety, and walking speed. Furthermore, it is of interest how well the BFT is tolerated by the person and whether it has an influence on the self-assessed postural stability in everyday life. The study is divided into a baseline measurement at time $\mathrm{T}_{0}$, a six week BFT, an intermediate test $\mathrm{T}_{1}$ three weeks after $\mathrm{T}_{0}$, and a final test $\mathrm{T}_{2}$ after a total of six weeks intervention period. The variables postural control, gait safety and walking speed were measured using the standard 10-Meter-Walking-Test (10MWT) and the Functional Gait Assessment (FGA).

\section{Measurements}

10-Meter-Walking-Test ${ }^{10-12}$ : A distance of 10 meters is passed six times, with a self-chosen recovery break being held between each lap. Within the first three rounds the patient is asked to walk in her pleasant walking speed. Within the second three rounds the patient is asked to walk as fast as possible (not running). The time of each round was stopped. Mean value of the first three rounds provides information on the pleasant and safe walking speed. Mean value of the last three rounds provides information of the possible maximum of gait speed. In order to get an overview of the real walking speed, the time from meter 2 to meter 8 is stopped so that acceleration and deceleration will be not included in the stopped time.

Functional Gait Assessment, German version ${ }^{13}$ The FGA is used to measure balance abilities in different walking tasks. Firstly described by Wrisley et al., ${ }^{14}$ the FGA represents the further development of the Dynamic Gait Index (DGI). ${ }^{15}$ Seven of the eight tasks out of the DGI are used in the FGA but changed in their operational definition. Additionally, three other tasks are included in the assessment. The
FGA consists of ten different items, which are rated on a scale from 0 to 3 points. The maximum score that can be achieved in the FGA is 30 points and indicates an unrestricted balance during walking.

\section{Intervention}

The intervention consisted of eleven treatment sessions (each session about 20 minutes, two sessions per week, one missed session in week three), carried out with a 4-channel surface EMG biofeedback amplifier (NeXus-10 MKII) and the software BioTrace + (Mindmedia B.V., Netherlands). For the training, the activity of the antagonistic stabilizing muscles was derived and reported back in form of real-time visual feedback via a screen in front of the subject. In each session, either the thigh muscles $(\mathrm{Mm}$. glutei medius et minimus and $\mathrm{Mm}$. adductores or the M. quadriceps femoris and the $\mathrm{Mm}$. ischiocrurales), or the lower limb muscles (M. gastrocnemius and M. tibialis anterior) were trained. The derivation took place on both sides of the same muscle groups. At the beginning, the perception of muscle control was visually supported by biofeedback. Subsequently, the stabilizing muscles should be deliberately tensed or relaxed by the participant, under constant feedback of the EMG signal. In addition, exercises (e.g. squats with the aim of symmetrical muscle activation) were applied. To increase the task difficulty, the weight should be shifted to one side and the appropriate co-contraction of the stabilizing muscles should be taken into account. The duration and breaks were adapted individually to the fatigue of the patient.

\section{Data analyses}

Due to the study design only a descriptive analysis is possible. Furthermore, a self-report of the participant will be added in order to enable information about the training tolerance and the personal experience in daily life.

\section{Results}

In Table 1 the results of the self-chosen walking speed ${ }^{\mathrm{m} / \mathrm{s}}$ and the maximum walking speed ${ }^{\mathrm{m} / \mathrm{s}}$ out of the 10MWT are shown. Specific changes of the maximum speed within the six weeks intervention period is demonstrated in the mean out of the three fast laps in all three testing points (Figure 1). While the needed time in the 10MWT has been reduced after six weeks (-6\% compared to baseline), the results of the FGA ( $\mathrm{T}_{0}: 22$ points, $\mathrm{T}_{1}: 20$ points, $\mathrm{T}_{2}: 24$ points) showed a decrease after the first three weeks of the intervention $(-10 \%$ compared to baseline). However, in $\mathrm{T}_{2}$ the postural stability increased, demonstrated by a higher FGA-Score ( $+9 \%$ compared to baseline) (Figure 2).

Table I Results of IOMWT in all three testing points $\left(\mathrm{T}_{0}, \mathrm{~T}_{1}, \mathrm{~T}_{2}\right)$

\begin{tabular}{lll}
\hline $\begin{array}{l}\text { Testing } \\
\text { Point }\end{array}$ & $\begin{array}{l}\text { Mean of the Self-Chosen Mean of the Maximum } \\
\text { Walking Speed }[\mathrm{m} / \mathrm{s}]\end{array}$ & Walking Speed $[\mathrm{m} / \mathrm{s}]$ \\
\hline T0 & 1.16 & 1.38 \\
TI & 1.16 & 1.47 \\
T2 & 1.15 & 1.49 \\
\hline
\end{tabular}

In summary, the participant tolerated the EMG-Biofeedback training very well. Although the work-out was lower dosed than in her well-known physiotherapeutic treatment, she had the feeling that the biofeedback training was more exhausting, compared to her regular physiotherapy. The "cuff feeling" around the ankle joints after prolonged standing or walking - she mentioned as a problem before the beginning of the study - has been vanished after the six-week intervention period. Additionally, she mentioned a better perception of her feet in the moment of foot contact while walking what gives her more secure feeling in everyday life. 
Qualitatively analyzed video data of the $10 \mathrm{MWT}$ at $\mathrm{T}_{0}, \mathrm{~T}_{1}$ and $\mathrm{T}_{2}$ confirmed higher gait stability, shown in less deviation to the side while walking in testing point $T_{2}$ compared to $T_{0}$ and $T_{1}$.

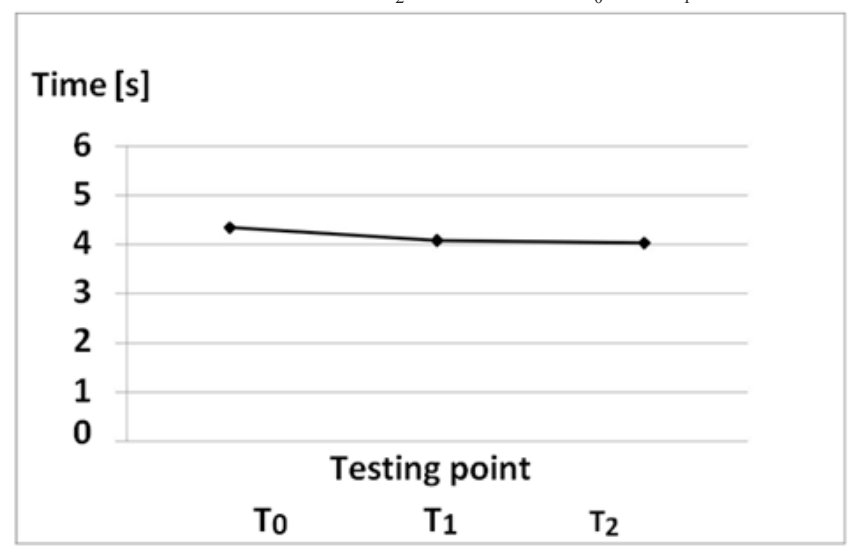

Figure I Results of the fastest speed in IOMWT in all three testing points $\left(\mathrm{T}_{0}, \mathrm{~T}_{1}, \mathrm{~T}_{2}\right)$.

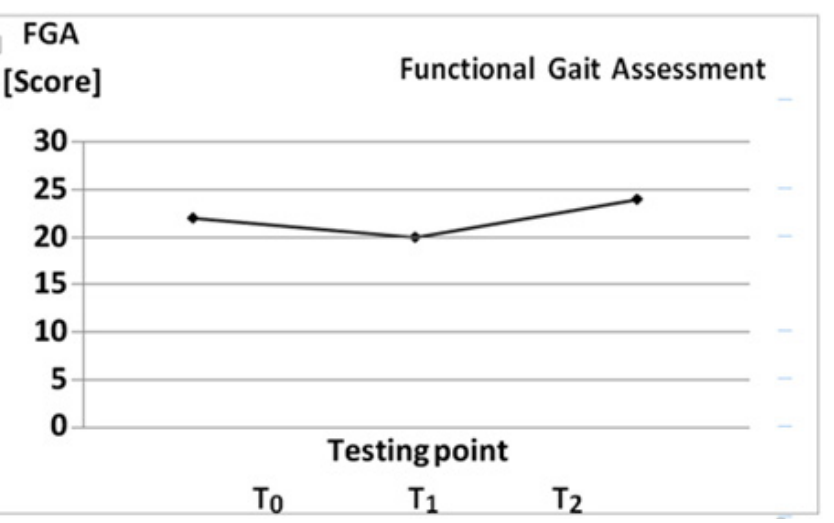

Figure 2 Results of the Functional Gait Assessment in all three testing points $\left(\mathrm{T}_{0}, \mathrm{~T}_{1}, \mathrm{~T}_{2}\right)$.

\section{Discussion}

The present case study gives indications of the effectiveness of EMG-supported Biofeedback training in a person suffering from Multiple Sclerosis regarding the improvement of postural control and gait. Because the Biofeedback treatment focuses on an improvement of (body) perception and an increased influence on physiological processes, especially the subjective information of the participant and the video data are important tools, next to quantitative assessments, in order to evaluate the therapy method.

Considering the results in the FGA, in a population of 60 to 96 -years old persons the Minimal Clinically Important Difference (MCID) is reported with four points. An increase in the FGA score about four points is interpreted as an additional value for the participant and is defined as clinically relevant. ${ }^{16}$

The participant of the present case study indicates a selfperceived improvement in gait stability, which has been confirmed in the qualitative evaluation of the video data. Additionally, the improvement of the FGA from $T_{1}$ to $T_{2}$ about four points indicates a clinically relevant improvement in balance while walking. The change in walking speed from $T_{0}$ to $T_{2}$ about $0.11 \mathrm{~m} / \mathrm{s}$ in maximum walking speed out of the 10MWT cannot be interpreted as clinically relevant or might be indicating a measuring error. Lang et al. ${ }^{17}$ have analyzed in persons suffering from Parkinson's Disease (PD) the Minimal Detectable Change (MDC) for the 10MWT. In the self- chosen walking speed the MDC amounts to $0.22 \mathrm{~m} / \mathrm{s}$ and in the maximum walking speed to $0.23 \mathrm{~m} / \mathrm{s}$. In our case, the subject did not receive those changes in walking speed, but we should keep in mind that she started with very good results at baseline. Bohannon ${ }^{10}$ gives norm data in healthy females over 70 years with a self-chosen walking speed about $1.27 \mathrm{~m} / \mathrm{s}$ and maximum walking speed of $1.74 \mathrm{~m} / \mathrm{s}$ an (compared to the baseline results of our case in $\mathrm{T}_{0}: 1.16 \mathrm{~m} / \mathrm{s}$ und 1.38 $\mathrm{m} / \mathrm{s}$ ). Therefore, it can be assumed that the results in the 10MWT might be seen as real improvements in the present case. Until now, EMG-supported biofeedback training has been applied and examined especially in order to improve the control in pelvic floor muscles in pwMS. ${ }^{8,18,19}$ In concern of gait and balance, biofeedback training has been applied in people with PD, because the usage of internal and external cues has been proved successfully in the therapeutic setting of persons suffering from this specific neurodegenerative disease. ${ }^{20-23}$ Internal cues are defined as body signals (e.g. EMG-derivation of single muscles), the body produces itself, whereas external cues are predefined from outside (e.g. metronome or a ladder on the ground). Even if the origin of the gait and balance disorders in persons with PD cannot be compared to those in MS, pwMS might benefit from biofeedback training as persons with PD actually do. Beyond, there are no side effects known in biofeedback training. ${ }^{5}$

\section{Conclusion}

The further development of current existing rehabilitation interventions is meaningful both in diseases with completely clarified pathogenesis, and in diseases without knowledge of all diseaserelevant processes in order to keep autonomy plus physiological, sensomotoric, psychological, and social functions of the affected people. The usage of goal-oriented physical exercise, training and physiotherapeutic treatments respectively, plays more and more an important role in neurorehabilitation. Biofeedback training provides a further non-invasive therapeutic approach in order to support pwMS in their current individual motor impairments and to improve their management of activities of daily living.

\section{Acknowledgements}

None.

\section{Conflict of interest}

None.

\section{Funding}

None.

\section{References}

1. Gehlen W. Neurologie. (12 ${ }^{\text {th }}$ edn), Thieme, Stuttgart, Germany. 2010.

2. Flachenecker P. Autoimmune diseases and rehabilitation. Autoimmunity reviews. 2012;11(3):219-225.

3. Multiple Sclerosis International Federation. Atlas of MS 2013. Zugriff. 2013.

4. Henze T, Rieckmann P, Toyka KV. Multiple Sclerosis Therapy Consensus Group of the German Multiple Sclerosis Society. Symptomatic treatment of multiple sclerosis. Multiple Sclerosis Therapy Consensus Group (MSTCG) of the German Multiple Sclerosis Society. European neurology. 2006;56(2):78-105.

5. German Society for Biofeedback e.V. Biofeedback. DGBfb e.V. 2017.

6. Jensen MP, Gianas A, George HR, et al. Use of Neurofeedback to Enhance Response to Hypnotic Analgesia in Individuals With Multiple Sclerosis. Int J Clin Exp Hypn. 2016;64(1):1-23. 
7. Choobforoushzadeh A, Neshat-Doost HT, Molavi H, et al. Effect of neurofeedback training on depression and fatigue in patients with multiple sclerosis. Appl Psychophysiol Biofeedback. 2015; 40(1):1-8.

8. Lúcio AC, D'Ancona CA, Lopes MH, et al. The effect of pelvic floor muscle training alone or in combination with electrostimulation in the treatment of sexual dysfunction in women with multiple sclerosis. Mult Scler. 2014;20(13):1761-1768.

9. Prosperini L, Leonardi L, De Carli P, et al. Visuo-proprioceptive training reduces risk of falls in patients with multiple sclerosis. Mult Scler. 2010;16(4):491-499.

10. Bohannon RW. Comfortable and maximum walking speed of adults aged 20-79 years: reference values and determinants. Age Ageing. 1997;26(1):15-19.

11. Bohannon RW, Andrews AW, Thomas MW. Walking speed: reference values and correlates for older adults. J Orthop Sports Phys Ther. 1996;24(2):86-90.

12. Wolf SL, Catlin PA, Gage K, et al. Establishing the reliability and validity of measurements of walking time using the Emory Functional Ambulation Profile. Phys Ther. 1999;79(12):1122-1133.

13. Thieme H, Ritschel C, Zange C. Functional Gait Assessment deutsche Version1 Übersetzung eines Instruments zur Beurteilung der Balancefähigkeit während des Gehens. Physioscience. 2009;5:5-11.

14. Wrisley DM, Marchetti GF, Kuharsky DK, et al. Reliability, internal consistency, and validity of data obtained with the functional gait assessment. Phys Ther. 2004;84(10):906-918.

15. Whitney S, Wrisley D, Furman J. Concurrent validity of the Berg Balance Scale and the Dynamic Gait Index in people with vestibular dysfunction. Physiother Res Int. 2003;8:178-186.
16. Beninato M, Fernandes A, Plummer LS. Minimal clinically important difference of the functional gait assessment in older adults. Physical Therapy. 2014;94(11):1594-1603.

17. Lang JT, Kassan TO, Devaney LL, et al. Test-Retest Reliability and Minimal Detectable Change for the 10-Meter Walk Test in Older Adults With Parkinson's disease. J Geriatr Phys Ther. 2016;39(4):165-170.

18. McClurg D, Ashe RG, Lowe-Strong AS. Neuromuscular electrical stimulation and the treatment of lower urinary tract dysfunction in multiple sclerosis--a double blind, placebo controlled, randomised clinical trial. Neurourol Urodyn. 2008;27(3):231-237.

19. Klarskov P, Heely E, Nyholdt I, et al. Biofeedback treatment of bladder dysfunction in multiple sclerosis. A randomized trial. Scand J Urol Nephrol. 1994;157(Suppl):61-65.

20. Lefaivre SC, Almeida QJ. Can sensory attention focused exercise facilitate the utilization of proprioception for improved balance control in PD? Gait Posture. 2015;41(2):630-633.

21. van den Heuvel MR, van Wegen EE, de Goede CJ, et al. The effects of augmented visual feedback during balance training in Parkinson's disease: study design of a randomized clinical trial. BMC Neurol. 2013;13:137.

22. Zalecki T, Gorecka-Mazur A, Pietraszko W, et al. Visual feedback training using WII Fit improves balance in Parkinson's disease. Folia Med Cracov. 2013;53(1):65-78.

23. Rossi-Izquierdo M, Ernst A, Soto-Varela A, et al. Vibrotactile neurofeedback balance training in patients with Parkinson's disease: reducing the number of falls. Gait Posture. 2013;37(2):195-200. 